\title{
Le Messianisme des premiers romans de la déportation acadienne
}

\author{
Robert Viau \\ Université du Nouveau-Brunswick (Fredericton)
}

\begin{abstract}
L a saga tragique de la déportation des Acadiens a exercé, et continue à exercer, une veritable fascination sur les romanciers francophones, qu'ils soient d'Acadie, du Québec ou de France. Parfois la déportation est le thème central d'un roman, parfois elle n'est qu'évoquée, mais il est évident que cet événement historique a impressionné et inspire les littéraires. Dans cet article, je souhaite aborder la problématique historique et messianique des premiers romans de la deportation: Jacques et Marie (1865-1866) de Napoléon Bourassa, Le Cap au diable (1862-1863) de Charles Deguise et Elle et Lui (1940) d'Antoine-J. Léger. ${ }^{2}$

Je ne crois pas qu'il soit nécesssaire de rappeler les événements historiques qui ont inspiré ces écrivains ; ils font partie de notre psyché collective. Cependant, il convient de rappeler que les livres d'histoire qui portent sur la déportation des Acadiens, et sur lesquels se sont basés les romanciers, sont, par essence, autre chose que la réalité de l'événement et sujets à caution. Les événements décrits sont choisis, inventés, puisque l'histoire par sa nature même est à la fois choix de documents, interprétation et rédaction. Comme l'explique Lucien Febvre :
\end{abstract}

Mais toute l'histoire est choix. Elle l'est, du fait même du hasard qui a détruit ici, et là sauvegardé les vestiges du passé. Elle l'est du fait de l'homme : des que les documents abondent, il abrège, simplifie, met l'accent sur ceci, passe l'éponge sur cela. Elle $l^{\prime}$ est du fait, surtout, que l'historien crée ses materiaux ou, si l'on veut, les recrée : l'historien, qui ne va pas rôdant au hasard à travers le passé, comme un chiffonier en quête de trouvailles, mais part avec, en tête, un dessein précis, un problème à résoudre, une hypothèse de travail à vérifier. ${ }^{3}$ 
Face à tout récit historique, le lecteur doit se demander quelle est l'autorité, la valeur, la portée de ce récit : l'historien est-il temoin ou simple écho d'une tradition orale ou écrite ? est-il sincère, impartial ? peut-il être impartial ? a-t-il quelque intérêt à modifier la véritê, à grossìr ou à diminuer les faits ? Le récit le plus sérieux et le plus sincère peut renfermer des faits inexacts, des détails incomplets. Il faut comparer les différents récits, voir si les faits en question cadrent avec d'autres faits connus, cadrent aussi avec les idées de l'époque, avec les moeurs et les institutions. Enfin, il faut chercher à dégager la manière propre de voir, de comprendre, de reconstruire de l'historien afin de déterminer comment il interprète le passé à la lumière de son présent.

$S^{\prime}$ il faut tenir en suspicion l'historien, que penser du romancier historique? Pour que le romancier historique puisse pleinement jouer son rôle, rédiger un récit qui captive le lecteur, il doit croire à ce qu'il écrit, l'intérioriser. Cependant, le romancier qui développe une certaine image de la déportation, s'inspire de ce qui lui paraît le plus riche (et donc le mieux propre à inspirer ses écrits) mais pas nécessairement de ce qui est le plus vrai. Ce qu'enseigne en effet la lecture des romans historiques, $c^{\prime}$ est la multiplicité des schèmes d'interprétation qui se dégagent des événements lointains. En fait, le romancier historique n'a pas vraiment le choix, il choisit, tranche et découpe, privilégie tels aspects plutôt que tels autres, car l'histoire vraiment totale des événements de 1755 le confronterait au chaos. Pour autant que le romancier aspire à la signification, il se condamne à choisir une région, une époque, un groupe d'hommes et des individus dans ces groupes, et à les faire ressortir. Le roman historique est donc à la fois partial et partiel.

L'intérêt du critique traditionnel a été trop souvent de mesurer l'écart entre les faits historiques et ce que nous présente le romancier. Citer les documents de première main, en discuter les diverses interprétations transformerait cet article en ouvrage historique et risquerait de nous faire perdre de vue le but premier de notre travail qui est de rechercher le message que transmet l'auteur dans son oeuvre, le but qu'il s'est fixé en rédigeant un roman. Ce qui suscite l'intérêt n'est pas la fidelité aux faits, mais l'idée que les romanciers se font des événements. Dans les romans à l'étude, nous chercherons a dévoiler le parti pris du romancier. Et dans les récits de la déportation, les romanciers sont excessivement subjectifs : ils proposent ou imposent une interprétation spécifique des faits. Dans ce cas précis, la véritable question ne porte pas sur le degré de « réalité historique » du roman, de référentialité historique, difficile à cerner et non pertinent à l'objet de notre étude, mais bien sur le sens historico- 
didactique explicite ou implicite de ces oeuvres basées sur des événements tragiques.

Il faudrait commencer par définir la position des auteurs qui ont écrit sur le " Grand Dérangement » sinon nous serions confronté au même problème que souligne Sartre lorsqu'il discute de l'écrivain réaliste : «Nous a-t-on assez dit qu'il « se penchait, » sur les milieux qu'il voulait décrire. Il se penchait ! Où était-il donc? En l'air ? " Le romancier historique n'est pas « en l'air, » et ce qu'il veut trouver dans l'histoire, c'est d'abord lui-même, « être historique a part entière qui justifie le passé à ses propres yeux beaucoup plus que le passé ne le justifie aux yeux des autres. ${ }^{5}$ Dans les romans étudiés les auteurs véhiculent le point de vue idéologique, propagandiste ou didactique, de la classe bourgeoise de l'époque, catholique et nationaliste. Cette restriction de champ élitiste présente nécessairement un événement historique comme la déportation des Acadiens sous un jour peu favorable et réduit le roman au statut d'illustration, de démonstration que propose le romancier ou son représentant. Histoire et point de vue sont donc indissociablement liés, mais de quelle démonstration s'agit-il ?

À la suite de l'échec de la rébellion de 1837 et de l'exil des chefs du parti patriote, une nouvelle idéologie peu à peu évince l'idéologie libérale et affirme le rôle prépondérant du clergé. S'inspirant du modèle de l'ultramontanisme européen, Mgr Bourget propose aux catholiques canadiens-français le modèle d'une société très hiérarchisée et dominée par l'autorité suprême du Souverain Pontife. Dès lors, les ultramontains seront partisans de l'alliance du trône et de l'autel comme en Europe (ce qui transposé au Canada signifie royalistes et fidèles à la couronne britannique), partisans des conservateurs et ennemis jurés des libéraux, des rouges et des idées nouvelles. Cette domination de la société par les éléments conservateurs sert l'ordre établi. Orateurs, journalistes et écrivains ne cessent de proclamer les vertus du peuple canadien-français, peuple choisi par Dieu.

En effet, selon la doctrine du messianisme compensateur, la divine providence a soustrait le Canada à l'influence de la France au moment où celle-ci s'apprêtait à commettre les erreurs de la Révolution française. Le Canada est devenu au XlXe siècle le refuge de la tradition royaliste française et de la religion catholique. Protégé de l'américanisme envahissant par l'Angleterre, le Canada reste à l'écart de la corruption des biens matériels et connaît la seule occupation vraiment saine, l'agriculture. En somme, qu'importe l'infériorité économique ou militaire, puisque ce qui compte avant tout, c'est la supériorité morale et 
intellectuelle, et qu'à ce point de vue les Canadiens français, pour reprendre une expression utilisée par plusieurs à cette époque, sont les "Athéniens d'Amérique » dont la mission est de convertir le Nouveau Monde. Ainsi l'idéologie officielle, propagée par des écrivains de droite, enseigne au peuple à ne pas écouter les agitateurs politiques mais à prier et à attendre. Cette idéologie qui, au Canada, prit racine dans le diocèse de Montréal, s'étendra dans tout le Québec et l'Acadie et pendant plus d'un siècle maintiendra son emprise.

En littérature, I'idéologie officielle rappelle à l'homme de lettres sa mission de promouvoir le bien, de magnifier la vertu et de combattre le vice, de « favoriser les saines doctrines, de faire aimer le bien, admirer le

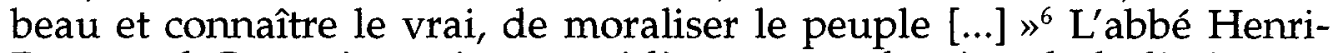
Raymond Casgrain, qui se considère comme le père de la littérature canadienne-française, écrit : " [notre littérature] sera grave, méditative, spiritualiste, religieuse, évangélisatrice comme nos missionnaires, généreuse comme nos martyrs, énergique et persévérante comme nos pionniers d'autrefois [...]. Mais surtout elle sera essentiellement croyante et religieuse. $\gg^{7}$

Dans un tel contexte, comment dépeindre dans un roman la tragédie de la déportation d'un peuple catholique et, surtout, comment réagir face à la barbarie britannique ? Ce qui surprend, à première vue, c'est que les premiers romans québécois et acadiens de la déportation suivent le même canevas. Dans Jacques et Marie, Le Cap au diable et Elle et Lui, les auteurs insistent tout $d^{\prime}$ abord sur l'honnêteté foncière des Acadiens. Ils démontrent, preuves à l'appui, que ceux-ci ont toujours été de fidèles sujets du roi d'Angleterre. Le jour de la convocation, les villageois se sont rendus à leur église sans aucune méfiance, " avec un esprit tout chevaleresque, se confiant à la loyauté anglaise " (CD 9). Ce sont les Anglais qui ont abusé de l'honnêteté des Acadiens pour les attirer dans un guet-apens et les déporter. Les auteurs décrivent tous le démembrement des familles, le départ des jeunes Acadiens qui, baïonnette au dos, $\mathrm{s}^{\prime}$ avancent en chantant des cantiques, les lamentations des femmes, les cris et les pleurs des enfants séparés de leurs parents, l'incendie des villages, la chasse à l'homme dans les bois... Ces auteurs cherchent surtout à attirer le blâme du lecteur sur les Anglais pour tant de souffrances imméritées.

Le jour de la déportation, les Anglais souillent leur propre réputation. Pour capturer plus facilement les Acadiens de Grand-Pré, ils les convoquent à l'église du village, sachant très bien que ces catholiques ne se douteraient de rien et n'oseraient croire en une perfidie perpétrée en 
ce lieu de culte. Ensuite, ils démembrent les familles lors de l'embarquement et surchargent les navires, ce qui va à l'encontre des ordres qu'ils ont reçus. À la veille de l'hiver, ils incendient les fermes et les champs afin d'affamer les Acadiens réfugiés dans la foret et lancent les Rangers à leur poursuite avec l'ordre de ramener les scalps de ces civils désarmés. Enfin, les misérables exilés qui reviennent des colonies américaines sont de nouveau refoulés vers le sud. Le sort des Acadiens pendant les années du Grand Dérangement est tragique ; pourtant, aux yeux des officiers britanniques, ce n'est que de la besogne bien faite. Pour charger encore davantage, les auteurs s'étendent amplement sur les causes dites "réelles » de la déportation (discrimination raciale et religieuse, cupidité) et sur le raffinement de cruauté des Anglais: massacre des Acadiens dans les bois, crimes inavouables contre des femmes acadiennes. enlèvement des enfants, etc.

Comment les personnages de ces romans réagissent-ils à ces outrages et quel enseignement l'auteur a-t-il à coeur de transmettre ? Dans Jacques et Marie, il y a tout d'abord la réaction vive du Micmac Wagontaga qui refuse de se soumettre, qui préfère combattre jusqu'à l'anéantissement, jusqu'à la dispersion de sa tribu. ${ }^{8}$ Mais l'auteur veille au grain ; Napoléon Bourassa, bien qu'il soit nationaliste, est avant tout ultramontain et les sentiments de ses Acadiens, fervents catholiques, seront différents de ceux du Micmac. Il faut pardonner, insiste l'auteur car " dieu le veut » (JM 356). Ainsi, fuyant les troupes anglaises qui ont incendié les établissements dans le Coudiac, Mme Hébert, « trop âgée pour tant marcher, pour tant souffrir » (car elle a vu deux de ses enfants assassinés par les Britanniques), meurt en priant « Dieu de ne pas punir les Anglais à cause de leurs cruautés " (JM 171). D'autre part, M. Hébert, qui a toujours nourri une haine profonde pour les Britanniques, « pardonne aux Anglais » (JM 357) avant de recevoir les derniers sacrements. En somme, comme l'écrit l'auteur, la prière et la résignation « élève[nt] un être au-dessus des injustices et des vengeances de notre monde, et lui communique[nt], au seuil de la vie, cette vertu de l'amour et du pardon qui commence l'éternité du ciel » (JM 250). De plus, comme l'explique M. Hébert : «C'est Dieu qui nous a vaincus tous les deux, mon bon Jacques, non pas les Anglais » (JM 354) et il revient à Dieu de venger les Acadiens et de punir les Lawrence, Murray, Winslow et Butler. Les Anglais ne resteront pas triomphants dans leur crime, car la justice divine vengera l'innocence des Acadiens. Avant de mourir, M. Hébert a une vision dantesque : il voit les responsables de la destruction de l'Acadie rôtir en enfer (voir JM 355). Somme toute, dans Jacques et Marie, le plus grand 
mérite des Acadiens lors de la déportation aura été d'avoir « triomphé du malheur en gardant une ame pure » (JM 31).

Dans Le Cap au diable du docteur Charles Deguise. M. Saint-Aubin, "par une malheureuse fatalité » (CD 10), arrive aux Mines pour y transiger les affaires de son commerce de la pêche à la morue le jour fixé par la proclamation pour la réunion des Acadiens. Il subit donc le sort réservé aux Acadiens de la région: saisie de ses biens, séparation de sa famille, expulsion... Dans cette atmosphère de fin de civilisation, le curé tente d'apaiser ses paroissiens en célébrant une dernière messe et en leur adressant quelques paroles de consolation et d'adieu. D'une voix brisée par la douleur, il leur rappelle que Dieu protège les innocents, que les affligés seront consolés et récompensés, et que la terre est un lieu d'exil et de misères mais le ciel, la vraie patrie des croyants :

Dieu seul connaît ce que l'avenir nous réserve à tous, mais rappelons-nous que nous avons au ciel un bras tout-puissant, qui saura déjouer les complots des méchants : que ceux qui pleurent seront consolés et qu'ils recevront avec usure la récompense des larmes qu'ils auront versées. Car qu'est-ce que la terre que nous habitons sinon un lieu d'exil et de misères; mais le ciel, voilà notre patrie, vers laquelle doivent tendre nos désirs et nos aspirations (CD 11).

En septembre 1755 il n'y avait plus de prêtres dans les villages acadiens de la Nouvelle-Écosse et les églises étaient vides. Cette entorse à la vérité historique s'explique par la visée didactique de l'auteur : la défense du peuple acadien et la justification de la doctrine chrétienne. Dans ce récit, il faut qu'il y ait des prêtres en Acadie afin que ceux-ci encouragent les Acadiens et fassent connaître la volonté de Dieu. De plus, parce que les Saint-Aubin ont maintenu leur foi chrétienne malgré les événements malheureux, à la fin du récit, de retour en Acadie, ils recevront plus qu'ils n'avaient perdu. Comme l'explique un prêtre, Dieu a rendu Lazare à ses soeurs, alors que tous le croyaient mort, et rendu avec usure à Job « ce qu'il croyait perdu pour toujours » (CD 42). Les Acadiens seront recompensés de la même façon, en autant qu'ils aient la foi. L'image du soleil momentanément obscurci par des nuages rend bien la pensée de Deguise au sujet du sort des Acadiens de la déportation : 
- Madame, il serait mal à vous de douter de la toute puissance et de la bonté de Dieu. La vie pour vous a été comme un de ces jours où le soleil se lève radieux et brillant pendant quelques instants, puis de sombres nuages viennent en cacher l'éclat pendant quelque temps ; après les avoir dissipés, vous voyez l'astre du jour reparaître plus brillant qu'auparavant. Peut-être, madame, votre vie en est-elle à cette dernière phase et les ombres épaisses qui l'ont obscurcie vont-ils [sicl se dissiper comme le soleil dissipe les nuages (CD 42).

Elle et Lui du sénateur Antoine-J. Léger est le premier roman historique sur la déportation écrit par un Acadien (né à Memramcook) et publié en Acadie (à Moncton). Elle et Lui dépeint la vie simple, honnête et religieuse des Acadiens et établit la suite des événements qui ont mené à leur déportation. Dans son roman, l'auteur cite des documents d'époque et divers historiens, et s'applique à prouver la loyauté de ses ancêtres à la couronne britannique en réfutant l'une après l'autre les accusations portées contre eux par des historiens canadiens-anglais dans la version anglaise de la déportation.

Le jour de la convocation, Jean croit que le gouverneur veut réunir les Acadiens pour régler la question de leurs titres de propriété. À la suite de la lecture des instructions du roi, Jean, se rendant compte de sa méprise, argumente en vain avec le colonel Winslow : «On a joué de notre honnêteté, en prenant des moyens indignes pour nous rassembler ici [...] vous avez la force de votre côté, mais le droit de justice est avec nous " (EL 111). Les esprits s'échauffent, quelques coups de feu éclatent ; au mlileu de ce tumulte, on entend un vieillard à cheveux blancs dire, la voix étouffée de sanglots : «[...] pardonnez-nous nos offenses comme nous pardonnons à ceux qui nous ont offensés... " (EL 111). Jean se calme et remet son sort entre les mains de Dieu. Les autres Acadiens l'imitent :

Je me suis laissé emporter trop vite. La prière que ce vieillard vient de réciter nous rappelle bien que le bon Dieu est là pour tout voir, pour tout juger et pour tout réparer. D'ailleurs, il est plus heureux de pardonner que d'avoir besoin de pardon, et les vaincus sont parfois plus grands que les vainqueurs (EL 111). 
Puis c'est l'expulsion, avec cette suite de détails horribles auxquels nous ont habitué les romanciers précédents. Jean, séparé de sa famille, voit les soldats incendier Grand-Pré. Sa femme, Jeanne, prisonnière dans la cale d'un navire, est secondée par la plus jeune et la plus faible de ses filles. Sur une mer démontée, le bébé malade de la fille aînée de Jean et Jeanne meurt. La mère du bébé meurt peu après et son cadavre devient la pâture des requins. La tragédie est complète ; pourtant, les Acadiens ne se révoltent pas.

Jean est déporté à Boston où il retrouve deux de ses filles, Madeleine et Blanche. Faible et sans moyens, il ignore le sort des autres membres de sa famille. L'État du Massachusetts édicte une loi qui permet d'engager les Acadiens contre leur gré et de mettre leurs enfants en servage : les filles de Jean lui sont enleveés et placées en servitude. Jean et ses compatriotes envoient pétitions sur pétitions au gouverneur et son conseil mais en vain. Laissant le Massachusetts pour le Maryland, avec les débris de sa famille, Jean retrouve sa femrne et quelques-uns de ses enfants. Après dix années de misère et de désespoir, tous entretiennent le désir de revoir leur pays. C'est à Madeleine que revient la tâche de veiller sur la famille et de la ramener chez elle. Madeleine, première version de Pélagie-la-Charrette, encourage son père et sa mère, ses frères et ses soeurs, et tous se joignent à un groupe d'exilés et cheminent vers l'Acadie. Plusieurs d'entre eux, dont Jeanne, meurent en route. À l'automne de 1766, la famille s'installe enfin à Jemseg, sur les ruines des établissements français. Jean, un vieillard faible et dépourvu, regarde autour de lui, comme un homme égaré dans la forêt. C'est Madeleine qui donne le signal de la reconstruction. Elle saisit une hache et se met à abattre un arbre car il faut, comme elle le dit si bien, « préparer notre lit » (EL 169), construire des habitations et assurer la survie de la race.

Pendant la guerre de l'Indépendance américaine, les Acadiens ne se révoltent pas. Jean se rend même auprès des Micmacs pour rétablir l'ordre et la paix, et protéger les Anglais qui habitent des fermes qu'ils ont volées aux Âcadiens. Les Micmacs se laisseront convaincre par « leurs courageux, mais trop crédules frères blancs " (EL 197). La couronne britannique, fidèle à elle-même, sert ensuite aux Acadiens la même récompense : leurs nouveaux établissements sont donnés aux Loyalistes. Jean et ses compatriotes, qui cultivaient depuis dix-sept ans les terres neuves à Jemseg, sont chassés une deuxième fois de leur foyer. Écrasé par ces malheurs, mais croyant toujours en la miséricorde de Dieu, Jean meurt à l'âge de quatre-vint-cinq ans, en se soumettant à la volonté du Créateur. 
Comment expliquer une telle vie de malheurs? Un missionnaire nous donne un indice :

Le missionnaire est mandé. Il vient. Il leur conseille de garder dans leurs malheurs la dignité calme qui convient aux peuples sages et forts.

- Dieu, dit-il, se cache souvent pour voir jusqu'où vont la perversité des méchants et la patience des justes (EL 194).

Somme toute, d'après Léger, le peuple acadien est un peuple de martyrs chrétiens qui a toujours su résister aux sentiments de vengeance en puisant dans l'enseignement évangélique, « la résignation qui fait supporter la vie sans murmure " (EL 133).

- Mon père, fit Jean, il est regrettable que l'on se souvienne du passé par haine; je l'admets, mais comment faut-il aimer ceux qui nous ont fait du mal?

- Seulement pour l'amour du bon Dieu (EL 186).

Tout est disposé dans ces trois romans pour mettre en valeur la grandeur et l'honnêteté des Acadiens par opposition à la lâcheté et la duplicité des Anglais. L'honnêteté des uns s'oppose à la perfidie des autres. Ce procédé manichéen amène le lecteur à prendre conscience de l'inferiorité morale des Anglais et à apprécier toute la supériorité morale des vaincus. Les romanciers soulignent aux héritiers de Lawrence et Winslow toute la honte qui s'est accumulée sur eux à la suite de la conduite de leurs ancêtres pendant la déportation. C'est un sujet de honte que d'être Anglais après le traitement infligé aux Acadiens. C'est là un premier châtiment. Selon la justice immanente, un crime tel que la déportation porte en lui-même sa propre punition. De génération en génération, les Anglais dévorés de remords devront reconnaître la supériorité de leurs victimes. ${ }^{9}$ Deuxième châtiment, celui-ci dans l'audelà ; les Acadiens auront le plaisir d'assister au supplice éternel de leurs bourreaux. En somme, l'éternité sera pour les Acadiens une longue revanche.

Que nous révèlent ces premiers romans sur la ligne de conduite que devraient adopter les Acadiens? Wagontaga et les Micmacs préfèrent l'anéantissement de la tribu à l'asservissement. Les commerçants et 
soldats huguenots choisissent l'assimilation. Refusant à la fois l'anéantissement et l'assimilation, tout en imposant une fin de nonrecevoir à l'occupant, les romanciers proposent surtout une troisième voie, médiane. Ils réactualisent un scénario en apparence anodin, le messianisme, afin que les Acadiens puissent survivre en tant que peuple, résister à l'occupant et mener le combat politique.

On pourrait prétendre que la croyance en une mission providentielle est une évasion facile devant les conditions tragiques de l'existence des Acadiens ou même que l'église, par son message d'attente et de résignation, assure sa survie et la reconnaissance et la protection de l'état au détriment du mieux-être de ses fidèles. Mais le messianisme est $\mathrm{d}^{\prime}$ abord une réponse à un temps de crise ; il convient davantage au besoin de sécurité d'un peuple en convalescence, que ce soit les Québécois après 1838 ou les Acadiens au XIXe siècle. La réaffirmation de 1'autorité religieuse rassure une population foncièrement conservatrice et catholique, alarmée par le bouleversement des institutions traditionnelles et par l'ébranlement des certitudes doctrinales de l'Ancien Régime. L'image d'une autorité sécurisante incarnée par l'Église constitue une réponse rassurante... du moins jusqu'en la première partie du XXe siècle encore tout imprégné des idées issues du renouveau catholique du milieu du siècle précédent.

Confrontés à la tragedie de la déportation et à la quasi-disparition des Acadiens, les romanciers mettent en valeur les croyances vitales du peuple acadien (celles qui fondent son identité), c'est-à-dire ses origines françaises et sa religion catholique. C'est à partir de ces éléments qu'ils élaborent une "résurrection acadienne " possible. Ainsi, à la suite du traumatisme de la déportation, les personnages acadiens projetent sur l'avenir leurs croyances catholiques qui portent sur le salut et, en particulier, la croyance en la régénération d'un monde profané par la fin de ce monde et l'avènement inexorable du royaume de Dieu quand les justes seront récompensés et les méchants punis. Une telle réaction n'équivaut pas à un refus hallucinatoire du réel. La société acadienne, telle que décrite, exploitée, assoiffée d'absolu et de justice sociale ne se contente pas de rêver à cet état de félicité sociale possible ; elle s'organise pour y accéder en se rassemblant autour de croyances religieuses qui transforment son désespoir en espérance et qui lui permettent de surmonter son état de prostration et $\mathrm{d}^{\prime}$ agir sur son destin ici et maintenant.

La prédication d'un message eschatologique purificateur du monde est le seul, d'après ces auteurs, capable de réunir les Acadiens de la déportation autour de nouvelles options, comme celle du peuplement 
du Nouveau-Brunswick, et de créer une solidarité parfaite comparable à celle de la communauté des premiers chrétiens. L'idéal collectif proposé par les romanciers consiste donc à réorganiser le désir du mieux être et de l'avoir plus autour du pôle attractif du retour aux origines française et catholique. Ce retour aux origines assure une cohésion extraordinaire au groupe et lui donne des raisons de vivre. Ces romans se veulent un encouragement aux Acadiens à poursuivre le combat pour la survivance en suivant l'exemple des Anciens : la fidelité aux origines est garante de la survie de l'Acadie:

Le dimanche ! les jeunes surtout viennent assister à la lecture. Souvent, il [Jean] laisse le livre pour discuter avec eux l'avenir du pays ; les exhorter à s'attacher à tout ce qui les entoure; leur montrer l'exemple entraînant de ceux qui bûchent, qui labourent, qui sèment, qui moissonnent ; mais il finit toujours par leur faire comprendre que leur sort dépend moins de l'intérêt du pays que de l'amour de la race. Il leur dit que le devoir et la prière doivent s'enchaîner, se soutenir pour conduire le chrétien vers sa grande fin surnaturelle, et que, par l'accord et l'union, les Acadiens finiront peut-être par obtenir la jouissance de leurs libertés civiles et politiques (EL 60).

Selon ces romanciers, les Acadiens doivent puiser dans le temps de la déportation les forces qui leur sont nécessaires pour se libérer, c'est-àdire construire le royaume de Dieu en la Nouvelle Acadie. La figure de l'Acadien souffrant, seul capable par sa condition malheureuse de sauver le peuple, s'inscrit dans une visée politique. Le messianisme fournit un instrument de combat contre l'occupant et projette sur le monde un optimisme déliberé : celui de participer à la fondation d'une société radicalement autre, qui s'oppose à l'ordre établi anglais, trop puissant, trop monolithique, du moins pour le moment. Le messianisme se présente donc comme un moyen de survie collective, nécessaire au début, qui dissimule une visée politique : la participation au pouvoir, sinon la reprise du pouvoir.

Terminons par une ultime question. Pourquoi déterrer continuellement cette vieille querelle ? Pourquoi entretenir le souvenir du malheur? En fait, il suffit que cet épisode historique s'éloigne de nous dans la durée, 
ou que nous nous éloignions de lui par la pensée, pour qu'il cesse d'être intériorisable et perde son intelligibilité. Comme le note Raymond Aron : "L'homme n'a vraiment un passé que s'il a conscience d'en avoir un. »" Le passé n'acquiert de consistance, voire d'existence, que par la pensée qui réfléchit sur lui. La stratégie de Bourassa, Deguise et Léger s'inscrit autour de la conception du temps et plus précisément de l'histoire. Ce qui pousse ces auteurs à prendre la plume, c'est en fait une veritable nostalgie, un regret au sens exact du terme, de ne pas arriver à avoir raison de l'histoire. Mais justement, parce que la réalité ne satisfait pas toujours l'homme, le roman devient possible ; l'imagination romanesque vient corriger l'histoire. Ces premiers romans s'articulent autour d'une croyance messianique qui apporte une réponse au pourquoi du drame des Acadiens, galvanise les laissés pour compte de la guerre de Sept Ans et indique la voie à suivre pour réacturaliser le vieux rêve français et catholique. Ce sont des ouvrages de défense et d'illustration. En rectifiant l'histoire de la déportation, ces romanciers mettent les Acadiens en état de repousser toute insulte. De plus, en recourant à l'histoire, ils survolent la situation présente et entrevoient une issue aux problèmes contemporains et un destin à la race acadienne. Plutôt que de languir dans l'observation d'un présent décourageant, les romanciers croient qu'il faut construire l'avenir avec des fragments du passé. La conquête morale se fera donc par le retour aux sources nationales et les Acadiens survivront à condition d'être fidèles aux vertus du passé.

\section{Notes}

${ }^{1}$ Version remaniée $\mathrm{d}^{\prime}$ une communication présentée au deuxième colloque annuel de l'Association des professeur-e-s de littératures acadienne et québécoise de l'Atlantique.

${ }^{2}$ Dans la liste qui suit, chaque titre est précédé du sigle par lequel il est désigné dans cet article, puis de la référence bibliographique de l'édition que nous utilisons. $C^{\prime}$ est toujours aux pages et au texte de ces éditions que se conforment les citations.

JM Napoléon Bourassa, Jacques et Marie : Souvenir d'un peuple dispersé (Montréal : Fides, [Nénuphar ; 52] 1976), $371 \mathrm{p}$.

CD Charles Deguise, Le Cap au diable: Légende canadienne (Ste. Anne de la Pocatière [sic] : Firmin H. Proulx, 1863), 45 p.

EL Antoine-J. Léger, Elle et Lui: Tragique idylle du peuple acadien (Moncton: L'Évangéline Ltée 1940), 203 p.

${ }^{3}$ Lucien Febvre, Combats pour l'histoire (Paris : A. Colin, 1953), p. 7. 

p. 10.

${ }^{4} J$ Jean-Paul Sartre, Situations II : Qu'est-ce que la littérature (Paris : Gallimard, 1948),

${ }^{5}$ Michel Jarrety, "Valéry : l'Histoire, écriture d'une fiction, " Poétique no 49 (février 1982), p. 76.

${ }^{6}$ Henry-Raymond Casgrain, cité par René Dionne, Anthologie de la littétature québécoise, vol. II, La Patrie littéraire (1760-1895) (Montréal : La Presse, 1978), p. 308309.

${ }^{7}$ Henry-Raymond Casgrain, cité par Maurice Lemire, " La littérature canadiennefrançaise au XIXe siècle, "Dictionnaire des oeuvres littéraires du Québec, tome 1, Des origines à 1900 (Montréal : Fides, 1980), p. XXIII.

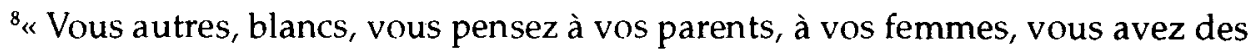
coeurs mous ; nous autres, nous ne voyons que l'insulte faite aux os de nos pères, et nous ne vivons pas s'ils ne sont pas vengés dans le sang de nos ennemis "(JM 337).

${ }^{9}$ Tout le roman $A u$ cap Blomidon porte sur le thème du remords qui poursuit les spoliateurs, de génération en génération, tant qu'ils n'auront pas réparé le tort causé aux Acadiens. Voir Alonié de Lestres [pseud. de l'abbé Lionel Groulx], Au cap Blomidon (Montréal : Granger frères, 1932), 239 p.

${ }^{10}$ Raymond Aron, Dimensions de la conscience historique (Paris : Plon, 1979), p. 11. 